\title{
Squashing and spaghettification in Newtonian gravitation
}

\author{
R. R. Machado*1@, A. C. Tort迎, C. A. D. Zarro² \\ ${ }^{1}$ Centro Federal de Educação Tecnológica Celso Suckow da Fonseca, Rio de Janeiro, RJ, Brasil \\ ${ }^{2}$ Universidade Federal do Rio de Janeiro, Instituto de Física, Rio de Janeiro, RJ, Brasil
}

Received on July 06, 2020. Revised on September 07, 2020. Accepted on October 08, 2020.

\begin{abstract}
Action films and sci-fi novels should be common resources in classrooms since they serve as examples and references involving physical situations. This is due to the abstract nature of many physical concepts, and the lack of references in students' daily lives makes them more difficult to be visualized or imagined. In this work we bring a discussion of how we can resort to films and literary works in order to elucidate the concepts regarding tidal forces. To this effect, we have used the action film Total Recall (2012) to present the squashing effect and the classic of juvenile literature From Earth to the Moon (1865) to discuss the effect of spaghettification in the context of Newtonian mechanics.
\end{abstract}

Keywords: Newtonian gravitation, non-inertial frames, tidal forces.

\section{Introduction}

Action movies and sci-fi novels can always be resorted to whenever we look for situations, or examples, capable of motivating our students. Total Recall is an action movie based on a story written by the American writer Philip K. Dick (1928-1982) and published in 1966 [1]. The first screen version of Total Recall was released in 1990, and the second one in 2012, see Figure 1. The 2012 version brings a new plot: The Earth has been devastated by a biological war and there were only two parts of the planet where humanity could live, namely, in a region of Europe comprising the British Isles and in a remote region in Australia. According to the new plot, in the region of Australia there is the so-called Colony, a kind of working-class suburban neighborhood where the people who work on robot assemblage factories go to rest and sleep. In The Colony, there is a designed special transportation named The Fall, see Figure 1] where workers would get a 17-minutes (sic) ride until their final destination. On board of The Fall the passengers are submitted to various physical effects - many of them discussed in an amusing video that can be watched in 2. Among those physical effects, one is particularly strange. There is a point at the center of the Earth where gravity is zero and as soon as one passes through this point, it inverts its direction. This point is unsurprisingly called the inversion point. But passengers on board of The Fall are most of the time in free fall, and as we will show, submitted to an effect that, though negligible from a practical point of view, nevertheless conceptually exists: The squashing.

Another interesting situation to be approached consists on Julio Verne's futuristic novel namely From the

\footnotetext{
${ }^{*}$ Correspondence email address: rodrigo.machado@cefet-rj.br
}

Earth to the Moon [3, see Figure 2, On Verne's chapter XXIII, he describes the Projectile-Vehicle, to which we will refer as Verne's projectile, designed to transport adventurous people through space; more precisely between the Earth and the Moon. Curiously in Verne's book we can also read about a special point to which the author refers as neutral point. This point of the journey is determined by the equality of the intensities of the gravitational attractions of the Earth and the Moon. In the book, the adventurers realize that they have reached that point when objects in the Verne's projectile playing the role of a spaceship start to float freely - exactly as in the 2012 remake of Total Recall when The Fall reaches the inversion point (the center of the Earth). As highlighted on the book [3]:

...But how should they know when the projectile had reached this neutral point situated at that distance, especially when neither themselves, nor the objects enclosed in the projectile, would be any longer subject to the laws of weight?

Up to this time, the travelers, while admitting that this action was constantly decreasing, had not yet become sensible to its total absence.

But that day, about eleven o'clock in the morning, Nicholl having accidentally let a glass slip from his hand, the glass, instead of falling, remained suspended in the air.

In Verne's projectile and in the The Fall, adventurers and workers, in their own reference frames, are in free fall, and unless they keep their seat belts fastened, everyone and everything floats freely. The remake of 2012 and the 19th century book are examples that can be 

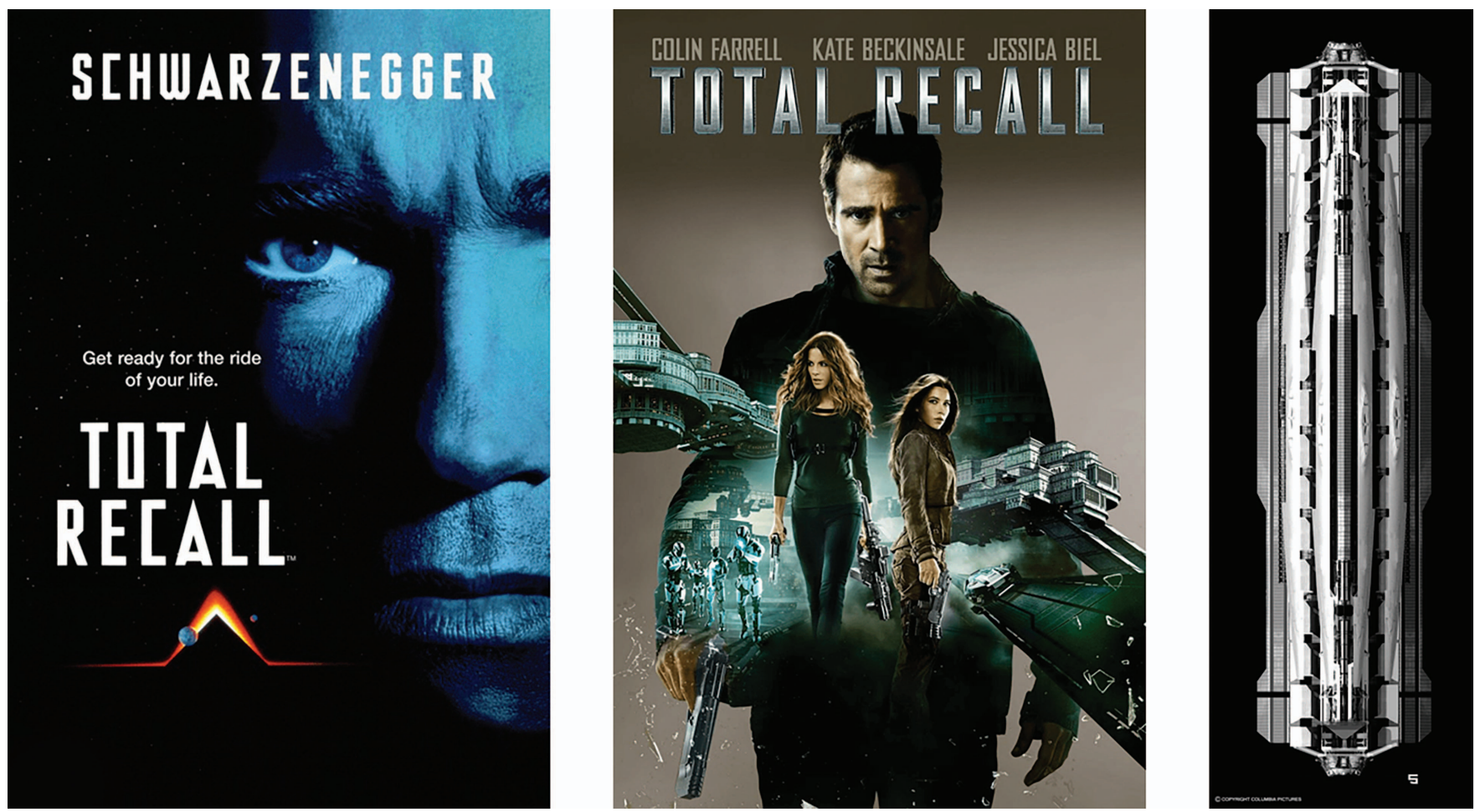

Figure 1: Left and center: Posters of the 1990 version and the 2012 remake. Right: The Fall. (Images Carolco Pictures and Columbia Pictures).
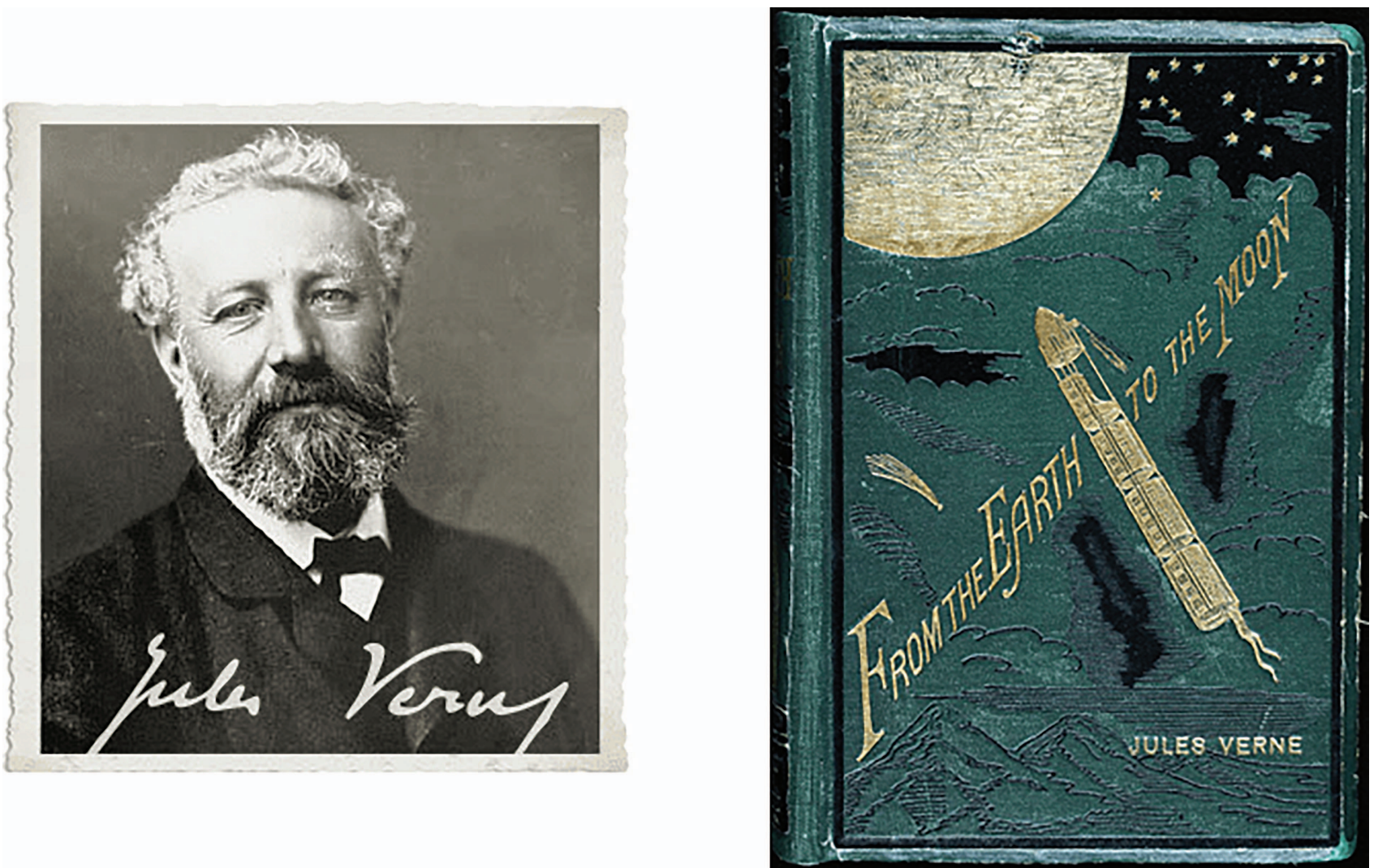

Figure 2: Jules G. Verne (1828-1905). On the right, cover of the first American edition of From the Earth to the Moon showing Verne's projectile/spaceship approaching the Moon. (Wikipedia Images) 
exploited in physics classes at undergraduate levels leading to a discussion on inertial and non-inertial reference frames in the context of Newtonian mechanics. In order to provide a better support for students and/or teachers, we have inserted a brief discussion on non-inertial reference frames.

\section{Non-inertial frames}

Newton's laws are valid for inertial frames of reference. A observer in an inertial frame of reference, say $S$, may verify the validity of the law of inertia (Newton's first law) on this frame. Thus, if the net force acting upon a body is null, the body will move on a straight line with constant velocity or will remain at rest in the inertial frame of reference. Besides, if Newton's laws are valid in $S$, they will also be valid for any other frames of reference moving at a constant velocity with respect to $S$.

Nonetheless, Newton's laws are not valid on a frame of reference $S^{\prime}$ accelerated with respect to an inertial frame of reference $S$, since for an observer in $S^{\prime}$, Newton's first law would not be verified on his reference frame. Since Newton's laws are not verified in this frame of reference, we name it non-inertial frame of reference. In the following, we will establish the equation of motion for noninertial frames of reference in pure translation. Let $S^{\prime}$ be a reference frame with an acceleration $\mathbf{A}$ with respect to $S$ (inertial) - see Figure 3 For the inertial frame $S$, the equation of motion for a particle of mass $m$ is given by Newton's second law:

$$
\sum \mathbf{F}=m \mathbf{a}
$$

where $\sum \mathbf{F}$ stands for the net force acting upon on a particle of mass $m$ and $\mathbf{a}$ is the acceleration of the particle with respect to $S$. For the accelerated frame $S^{\prime}$, we

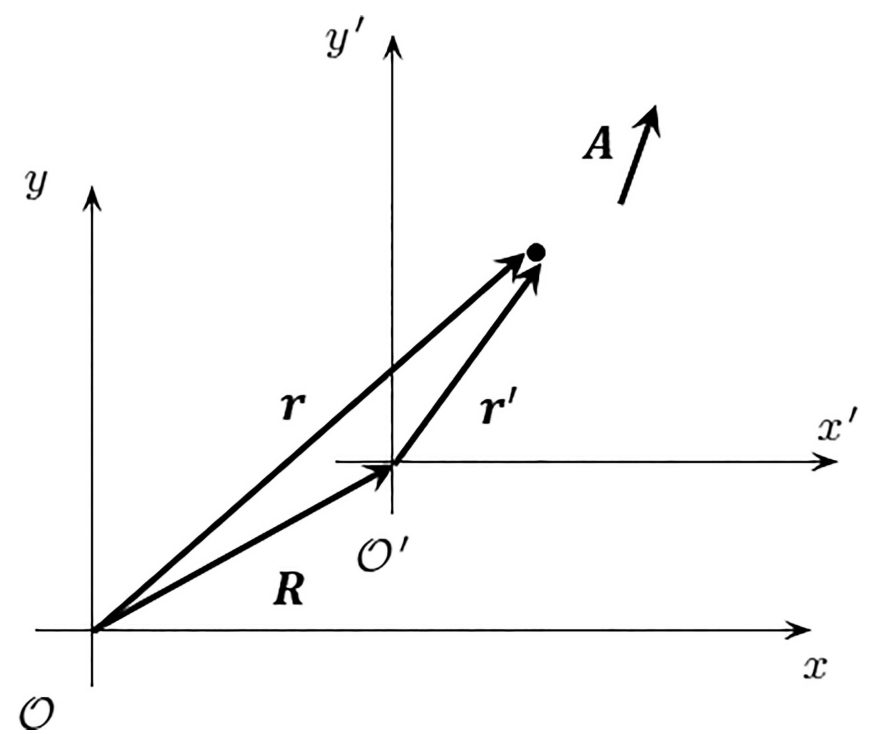

Figure 3: The frame of reference $S^{\prime}$ with an acceleration A with respect to the frame of reference $S$. cannot apply Newton's second law directly for a particle of mass $m$. Thus, we need to determine the corresponding equation of motion for in the non-inertial frame $S^{\prime}$. Examining Figure 3 , we can write the following expression for the position vectors $\mathbf{r}$ and $\mathbf{r}^{\prime}$ of a particle of mass $m$ with respect to $S$ and $S^{\prime}$, respectively:

$$
\mathbf{r}^{\prime}=\mathbf{r}-\mathbf{R}
$$

where $\mathbf{R}$ represents the instantaneous position of the origin for $S^{\prime}$ with respect to $S$. If we determine the second derivative with respect to time for the equation (1) we can obtain the relation between the acceleration $\mathbf{a}$ and $\mathbf{a}^{\prime}$ of the particle with respect to $S$ and $S^{\prime}$, respectively

$$
\mathbf{a}^{\prime}=\mathbf{a}-\mathbf{A}
$$

where A stands for the acceleration of $S^{\prime}$ with respect to $S$. If $S^{\prime}$ moves with constant velocity with respect to $S$ the term $\mathbf{A}=\frac{d \mathbf{V}}{d t}$ is null and the accelerations measured in $S$ and $S^{\prime}$, are identical. Multiplying equation (3) by the mass of the particle, we obtain:

$$
m \mathbf{a}^{\prime}=m \mathbf{a}-m \mathbf{A} .
$$

Since a represents the acceleration of the particle in $S$, an inertial frame of reference, we can apply Newton's second law and rewrite equation (3) as follows:

$$
m \mathbf{a}^{\prime}=\sum \mathbf{F}-m \mathbf{A} .
$$

Equation (4) represents the equation of motion for a particle of mass $m$ in a non-inertial frame $S^{\prime}$. The extra term $-m \mathbf{A}$ in equation (4) is often called inertial force. Thus, the inertial force can be written as

$$
\mathbf{F}_{\text {inertial }}=-m \mathbf{A} \text {. }
$$

If we take a look at equation (5), we will see that the inertial force has the same orientation, but the opposite direction to the acceleration of frame $S^{\prime}$ with respect to $S$. Observe that the inertial force is only present in the non-inertial frame of reference, being the 'force' we can feel whenever we are standing on a bus when it suddenly stops. The inertial forces are also known as pseudo forces or fictitious forces due to the fact that they are not exerted by an agent. In addition, the inertial forces also do not obey Newton's third law.

\section{Tidal forces}

Essentially, tidal forces are due to the non-uniformity of the gravitational field acting on an extensive body. In general, we associate tidal forces with the effect caused by the gravitational action of the Sun and Moon on Earth. Thus, due to the non-uniformity of the external gravitational field acting on the Earth, we can perceive deformations that are commonly called tides in 


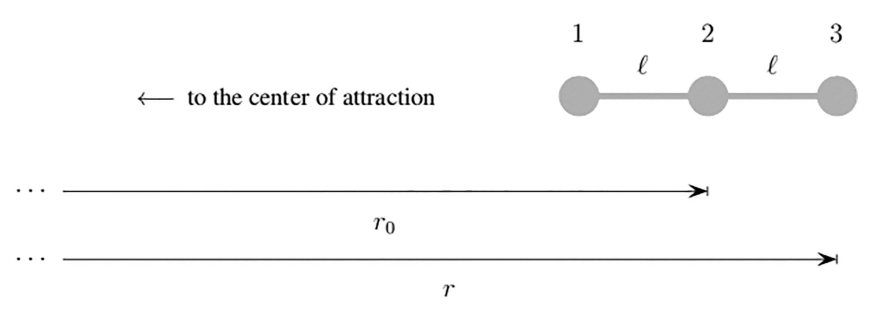

Figure 4: System of three particles under the exclusive action of a non-uniform gravitational field $\mathrm{g}$.

our oceans. However, tidal forces (which cause these deformations) can exist between any two extensive bodies. Thus, following Silveira 4 we will introduce tidal forces by analyzing an idealized system composed of three particles (1, 2 and 3 ), each of mass $m$, connected by light and rigid rods of length $\ell$. The system composed of the three particles will be under the exclusive action of a non-uniform external gravitational field $\mathbf{g}$ generated by say a planet - as illustrated in Figure 4 . The planet, or any mass whatsoever, is supposed to be spherically symmetrical of radius $R$ and mass $M$.

The gravitational field $\mathbf{g}$ for a mass distribution $M$ spherically symmetrical can be generally written in terms of its radial component as follows

$$
g_{r}=-G M f(R, r),
$$

where $\mathrm{G}$ stands for the gravitational constant and the function $f(R, r)$ is a generical function expressing the variation of the gravitational field with the radial coordinate $r$ and the radius of the planet, $R$. It can be shown with the help of Gauss' law that for a spherically symmetrical and homogeneous mass $M$ the gravitational field in its interior, $0 \leq r \leq R$, varies linearly with radial distance $r$ and in its exterior, $r \geq R$, the gravitational field varies according to the inverse-square law with respect to the center of the gravitational pull. Thus, the function $f(R, r)$ can be written

$$
f(R, r)=\left\{\begin{array}{l}
r / R^{3} ; \quad \text { for } r \leq R \\
1 / r^{2} ; \quad \text { for } r \geq R .
\end{array}\right.
$$

The gravitational force acting on a mass $m$ in a gravitational field $\mathbf{g}$ can be determined by

$$
\mathbf{F}_{\text {grav }}=m \mathbf{g} .
$$

Making use of Newton's second law, we can write the radial component of the acceleration of the center of mass of the system as

$$
a_{r_{\mathrm{C}}}=-G M f\left(R, r_{0}\right),
$$

where the radial coordinate $r_{0}$ locates the center of mass of the system. We can write the radial component of the gravitational force acting on the $i-$ th particle located at $r$ as

$$
\left(F_{r_{\text {grav }}}\right)_{i}=-G M m f(R, r)=-G M m f\left(R, r_{0}+\Delta r_{i}\right),
$$

where the index $i$ varies from 1 to 3 and $\Delta r_{1}=r_{0}-\ell$, $\Delta r_{2}=0$ and $\Delta r_{3}=r_{0}+\ell$. From equation (6) we see that the gravitational force is different on each one of the masses, but if the rods are rigid, the accelerations of masses 1, 2, and 3 have the same value for the radial component. As a result, if the net force is the same on each of the masses, the rods connecting particles 1,2 , and 3 must be submitted to tensions in such a way that the net force acting on each of the particles is the same. Hence, the simplified system presented in Figure 4 when placed in a non-uniform gravitational field is submitted to internal forces, i.e., tensions in the rods that connect masses 1, 2, and 3. Let us suppose now that the rods are flexible. In this case, the internal forces will cause a deformation in the system during the free fall in the gravitational field.

In Section 2 we have seen that it is possible to analyze a mechanical problem from the point of view of a non-inertial reference frame. However, by doing so, we must take into account the action of the inertial forces in our analysis. Thus, we can analyze the previous problem with respect to a reference frame $S^{\prime}$ comoving with the system during the free fall, that is, under the sole action of an external gravitational field. With respect to $S^{\prime}$ the inertial forces acting upon particles 1, 2 and 3 will be given by

$$
F_{r_{\text {inertial }}}=-m A_{r}
$$

where $A_{r}$ is the radial component of the accelerated reference frame $S^{\prime}$ with respect to a reference frame fixed at the center of the gravitational pull. The component $A_{r}$ of the acceleration of reference frame $S^{\prime}$ with respect to $S$ coincides with the acceleration of the center of mass of the particle system. From this, it follows that

$$
A_{r}=-G M f\left(R, r_{0}\right)
$$

Therefore, once more following Silveira 4, we can provide an operational definition of the tidal force in the accelerated reference frame $S^{\prime}$. It can be defined as 'the net of the gravitational force due to the external gravitational field with the inertial force in the reference frame accelerated by such external field.' Mathematically it reads

$$
\mathbf{F}_{\text {tidal }}=\mathbf{F}_{\text {inertial }}+\mathbf{F}_{\text {grav }} \text {. }
$$

Equation (7) can be applied at any point of the threeparticle system. In this sense, the tidal force in a mass $m$ located at $r$ coordinate, in terms of radial component, is written as

$$
F_{r_{\text {tidal }}}=-G M m f(R, r)-m A_{r} .
$$

Since $A_{r}=-G M f\left(R, r_{0}\right)$, it follows that

$$
F_{r_{\text {tidal }}}=-G M m f(R, r)+G M m f\left(R, r_{0}\right)
$$


Analyzing the previous relation, we find that the tidal force on mass 2, which coincides with the center of mass of the system, is null, that is

$$
F_{r_{\text {tidal }}}=-G M m f\left(R, r_{0}\right)+G M m f\left(R, r_{0}\right)=0 .
$$

Making use of equation (8) we can determine the tidal force on particle 1 and on particle 3 . The tidal force acting on particle 2 is zero, but the tidal force is different from zero for particles 1 and 3 . The tidal force $\mathbf{F}_{\text {tidal }}$ will point towards or away from the attraction center, this will be determined by $f(R, r)$ as we will see next in the case of The Fall and of Verne's projectile.

\section{Free falling reference frames and tidal forces}

The Fall and Verne's projectile are good examples of frames of reference in free fall. A frame of reference in free fall accelerates in the direction of the gravitational field at a certain location with magnitude $|\mathbf{g}|$. An observer in free fall in his own reference frame would have a feeling of having no weight, called weightlessness, and would fluctuate. By this, we can infer that the workers on board of The Fall and the adventurers of Verne's projectile would feel weightless all the time. The Fall and Verne's projectile are bodies in a non-homogeneous gravitational field, hence under the action of tidal forces.

We will analyze on sections 4.1 and 4.2 the situations for The Fall and Verne's projectile according to the free-falling frame of reference instantaneously co-moving with The Fall and Verne's projectile.

\subsection{The Fall}

In the free falling reference frame instantaneously comoving with The Fall, the tidal forces that act on a body of inertial mass $m$ released at a radial distance $r$ from the center of the Earth come from the combined action of the inertial force and the force of the gravitational attraction (see Figure 5), that is

$$
\mathbf{F}_{\text {tidal }}=\mathbf{F}_{\text {inertial }}+\mathbf{F}_{\text {grav }}=-m \mathbf{A}+\mathbf{F}_{\text {grav }},
$$

where A stands for the acceleration of the free-falling reference frame. The inertial force $\left(\mathbf{F}_{\text {inertial }}\right)$ must be taken into account due to the fact that we are considering the problem from the point of view of free-falling reference frame co-moving with The Fall. The magnitude of the

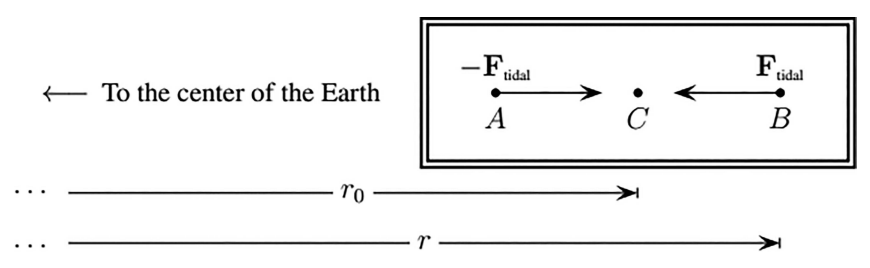

Figure 5: Tidal forces in the reference frame of the The Fall. inertial force acting on a test body of mass $m$ is equal in magnitude to the product of the mass by the acceleration $\mathbf{A}$, but as a vector it points to the opposite sense of the reference frame acceleration with respect to the Earth, as discussed in Section 2 .

In the case of the gravitational force, if we consider the Earth as a perfect homogeneous sphere, the gravitational field in its interior varies linearly with radial distance $r$ from the center of the Earth. The exact expression for the gravitational force, in terms of the radial component, reads

$$
F_{r_{\text {grav }}}=-\frac{G M m}{R_{\oplus}^{3}} r=-\frac{G M m}{R_{\oplus}^{3}}\left(r_{0}+\Delta r\right)
$$

where $G$ is the gravitational constant, $r_{0}$ is the distance between the center of the Earth and the center of mass of The Fall, $\Delta r=r-r_{0}$ is the radial displacement from $r_{0}$, $R_{\oplus}$ and $M$, are the Earth's radius and mass, respectively. For a test body placed at the center of mass of The Fall, the tidal force (equation 7 ) is zero and we can write the following relation

$$
-m A_{r}=+\frac{G M m r_{0}}{R_{\oplus}^{3}}
$$

where $A_{r}$ stands for the radial component of the acceleration of The Fall. Notice that we are not taking into account the convergence of the lines of force of the gravitational field as the observer approaches the center of the Earth. In other words, we are assuming that the transversal dimension of The Fall is negligible in a first approach. For $r \neq r_{0}$, the radial component of tidal force will be given by

$$
\begin{aligned}
F_{r_{\text {tidal }}} & =\frac{G M m r_{0}}{R_{\oplus}^{3}}-\frac{G M m}{R_{\oplus}^{3}} r_{0}\left(1+\frac{\Delta r}{r_{0}}\right) \\
& =-\frac{G M m}{R_{\oplus}^{2}} \frac{\Delta r}{R_{\oplus}} .
\end{aligned}
$$

Notice that this result is exact. The algebraic sign of the tidal force depends on the algebraic sign of $\Delta r$. If $r>r_{0}$ (point B in Figure 5), the tidal force points towards the center of the Earth; if $r<r_{0}$ (point A in Figure 5), the tidal force will point outwards, and The Fall will be under the effect of squashing. If $\Delta r=0$, the tidal force will be zero and a test body placed in the middle of the wagon will be in free fall. Tidal forces cannot be eliminated by a convenient change of reference frame as shown in an unequivocal way with The Fall. If a passenger is not in the central part of The Fall, point $C$ in Figure 5 she or he will be under the action of tidal forces. Let us suppose, for instance, that $\Delta r$ is of the order of $100 \mathrm{~m}$. In this case, since the radius of the Earth is of the order of $10^{6} \mathrm{~m}$, the ratio $\Delta r / R_{\oplus}$ is $10^{-4}$ and the tidal force on a, say, $70 \mathrm{~kg}$ passenger would be $7 \times 10^{-1} \mathrm{~N}$, small, but nevertheless measurable. We can also imagine a special sledge with room for just one passenger. Let us suppose, again, that the sledge has a length equal 
to $2 \mathrm{~m}$ and it goes down the tracks towards the center of the Earth. Then at her/his feet the passenger will feel a force towards the center of the sledge and the same will occur at her/his head. In other words, the rider will be squashed not stretched.

\subsection{Verne's projectile}

In the case of Verne's projectile, the evaluation of the tidal force follows an analogous reasoning to the previous one. The difference is that now the gravitational field varies according to the inverse-square law. Besides, the forces of attraction of the Earth and the Moon on a test body can be computed. With respect to an free-falling reference system co-moving with the projectile, the tidal force on a test body placed at $r$ reads

$$
\mathbf{F}_{\text {tidal }}=\mathbf{F}_{\text {inertial }}+\mathbf{F}_{\text {grav }}=-m \mathbf{A}+\mathbf{F}_{\text {grav }}
$$

where in terms of the radial component

$$
\begin{aligned}
F_{r \text { grav }} & =-\frac{G M_{\oplus} m}{r^{2}}+\frac{G M_{\text {moon }} m}{(D-r)^{2}} \\
& =-\frac{G M_{\oplus} m}{\left(r_{0}+\Delta r\right)^{2}}+\frac{G M_{\text {moon }} m}{\left[D-\left(r_{0}+\Delta r\right)\right]^{2}} .
\end{aligned}
$$

Here $M_{\text {moon }}$ is the mass of the Moon, $D$ is the distance between the centers of the Earth and the Moon, and $\Delta r=r-r_{0}$, see Figure 6 As before, the inertial force is $\mathbf{F}_{\text {inertial }}=-m \mathbf{A}$, where now at the center of mass of the Verne's projectile we have

$$
-m A_{r}=+\frac{G M_{\oplus} m}{r_{0}^{2}}-\frac{G M_{\text {moon }} m}{\left(D-r_{0}\right)^{2}} .
$$

Therefore, in the projectile reference frame, the tidal force, in terms of the radial component, will be given by

$$
\begin{aligned}
F_{r \text { tidal }}= & -m A_{r}+F_{\text {gravitational }} \\
= & \frac{G M_{\oplus} m}{r_{0}^{2}}-\frac{G M_{\text {moon }} m}{\left(D-r_{0}\right)^{2}}-\frac{G M_{\oplus} m}{\left(r_{0}+\Delta r\right)^{2}} \\
& +\frac{G M_{\text {moon }} m}{\left[D-\left(r_{0}+\Delta r\right)\right]^{2}} ;
\end{aligned}
$$

which, assuming that $\Delta r / r_{0} \ll 1$ and $\Delta r /\left(D-r_{0}\right) \ll 1$, and making use of the approximation $(1+x)^{n} \approx 1+n x$, equation 12 can be rewritten as

$$
F_{r \text { tidal }} \approx \frac{2 G M_{\oplus} m}{r_{0}^{2}} \frac{\Delta r}{r_{0}}+\frac{2 G M_{\text {moon }} m}{\left(D-r_{0}\right)^{2}} \frac{\Delta r}{D-r_{0}}
$$

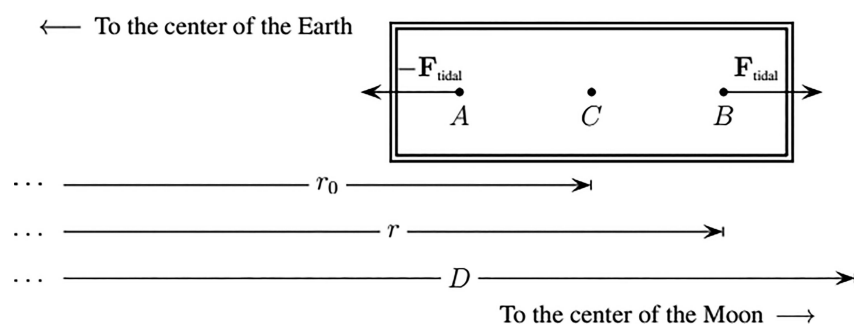

Figure 6: Tidal forces in the reference frame of Verne's projectile.
Introducing the adimensional variable $x=r_{0} / D$, the above equation reads

$$
F_{r_{\text {tidal }}} \approx \frac{2 G M_{\oplus} m \Delta r}{D^{3}}\left[\frac{1}{x^{3}}+\frac{M_{\text {moon }} / M_{\oplus}}{(1-x)^{3}}\right] .
$$

Using $\Delta r=100 \mathrm{~m}, m=70 \mathrm{~kg}, D=3,84 \times 10^{8} \mathrm{~m}$ and $M_{\oplus}=5,79 \times 10^{24} \mathrm{~kg}$, one has

$$
\frac{2 G M_{\oplus} m \Delta r}{D^{3}}=9,85 \times 10^{-8} \mathrm{~N}
$$

and

$$
\frac{M_{\text {moon }}}{M_{\oplus}}=0,01
$$

The range of Verne's projectile is $\left[x_{\min }, x_{\max }\right]$, where

$$
\begin{aligned}
& x_{\min }=\frac{R_{\oplus}}{D}=0,02, \\
& x_{\max }=1-\frac{R_{\text {moon }}}{D}=0,99 ;
\end{aligned}
$$

where $R_{\oplus}=6,37 \times 10^{6} \mathrm{~m}$ and $R_{\text {moon }}=1,74 \times 10^{6} \mathrm{~m}$ is the radius of the Moon. Hence, the tidal force in Verne's projectile is

$$
F_{\text {ridal }}=9,8546 \times 10^{-8}\left(\frac{1}{x^{3}}+\frac{0,0123}{(1-x)^{3}}\right),
$$

in the range $0,02 \leq x \leq 0,99$. In this region, the tidal force forces for $r_{0}=R_{\oplus}$ and $r_{0}=D-R_{\text {moon }}$ are

$$
\begin{aligned}
F_{r_{\text {tidal }}}\left(R_{\oplus} / D\right) & \approx 0,22 \times 10^{-1} \mathrm{~N}, \\
F_{r_{\text {tidal }}}\left(1-R_{\text {moon }} / D\right) & \approx 0,13 \times 10^{-1} \mathrm{~N} .
\end{aligned}
$$

The plot of the tidal force is depicted in Figure 7 Its minimum occurs at $x_{\min }=0,75$, which means that $r_{0}=0,75 D=2,87 \times 10^{5} \mathrm{~km}$, where the tidal force is

$$
F_{r_{\text {tidal }}}\left(x_{\text {min }}\right) \approx 3,11 \times 10^{-7} \mathrm{~N} .
$$

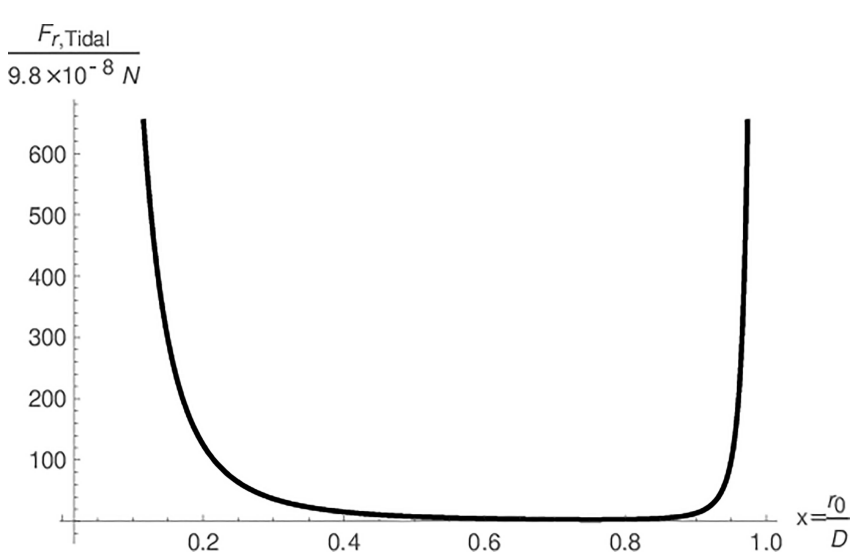

Figure 7: Plot of the tidal force in units of $\frac{2 G M_{\oplus} m \Delta r}{D^{3}}$ versus the adimensional variable $x$. For a long range of values in Verne's Fall, the observer will measure a negligible tidal force. Even near the surfaces of the Earth and Moon, the force is very tiny. 


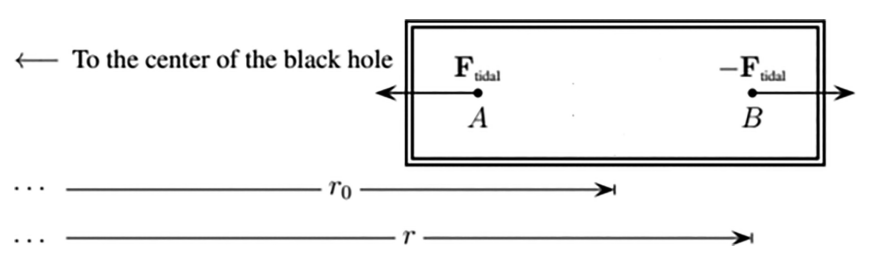

Figure 8: Tidal forces in the free falling reference frame into a non-rotating black hole.

As in the previous case, the direction of the tidal force (equation 13) depends on the algebraic sign of $\Delta r$. If $r>r_{0}$, as at point $B$ in Figure 6, the tidal force will point to the center of the Moon; but if $r<r_{0}$ as at point $A$ in Figure 6, the tidal force will point to the center of the Earth. This effect is known as "spaghettification".

\section{Falling into a non-rotating black hole}

It can be show that for a purely radial motion into or out of a black hole, the Newtonian tidal acceleration formulae that we derived also holds in the case of a nonrotating black hole or a Schwarzschild black hole of mass $M_{\text {black hole }}$ [5, 6]. Let us consider equation (13) and replace the Earth by the non-rotating black hole, let $D \rightarrow \infty$, and divide the result by the mass $m$. It follows that

$$
a_{\text {tidal }} \approx \frac{2 G M_{\text {black hole }}}{r_{0}^{2}} \frac{\Delta r}{r_{0}} .
$$

Again, if $\Delta r>0$ (point $\mathrm{B}$ ), the tidal acceleration and forces will point radially outwards; and if $\Delta r<0$ (point A), they will point radially inwards, see Figure 8

For a stellar black hole with a mass of, say, 3.5 solar masses, that is, $M_{\text {black hole }}=3.5 M_{\odot}$, we obtain $M_{\text {black hole }}=6,96 \times 10^{30} \mathrm{~kg}$. If now we set $r_{0}$ to equal to the Schwarzschild radius 1 we obtain $a_{\text {tidal }} \approx 9 \times$ $10^{10} \mathrm{~m} / \mathrm{s}^{2}$. The projectile (in Figure 6 ) will be subjected, again, to the effect of "spaghettification", however in this case the stretching forces will be unimaginably strong.

\section{Final remarks}

The situation described in the action movie Total Recall and in the juvenile literature From the Earth to the Moon allows us to discuss the physics of non-inertial frames of reference. Due to the non-uniform gravitational field acting upon The Fall and Verne's projectile there is the action of tidal forces. For The Fall, whose gravitational field has a linear variation with the radial distance, we observe the occurrence of the squashing effect. While for

\footnotetext{
1 In a simplified way, the Schwarzchild radius can be defined as the radius in which for a particle escaping the gravitational pull generated by the planet, of mass $M$, it would be necessary that its escape speed were equal to the speed of light. The result is $R s=2 G M / c^{2}$. Incidentally, this classical reasoning enables to obtain the very value as found in general relativity.
}

Verne's projectile, whose gravitational field varies with the inverse-square law, we observe the occurrence of the spaghettification effect. Lastly, we have observed that for a spaceship in the vicinity of a black hole, it would suffer the action of incredibly intense forces leading to the spaghettification effect. We believe that the examples discussed in this work can be used with a pedagogical profit to introductory and intermediate courses on classical mechanics.

\section{Acknowledgements}

The authors are grateful to Dr. Mariana Francisquini and the referees for their helpful comments which doubtlessly improved the final form of our manuscript.

\section{References}

[1] P.K. Dick, in: The Philip K. Dick Reader (Carol Publishing Group, Secaucus, 1997).

[2] Reel Physics, Total Recall-The Fall, available in: https://youtu.be/TmCmMmT7OX4.

[3] J. Verne, From the Earth to the Moon (Illustrated 1874 Edition) (SeaWolf Press, Orinda, 2019).

[4] F.L. Silveira, Caderno Brasileiro de Ensino de Física 20, 10 (2003).

[5] E.F. Taylor, J.A. Wheeler and E. Bertschinger, Exploring Black Holes: Introduction to General Relativity, available in: http://www.eftaylor.com/exploringblackholes/

[6] C.H. Misner, K.S. Thorne and J.A. Wheeler, Gravitation (Freeman, San Francisco, 1970). 